MetricForensics: A Multi-Level Approach for Mining Volatile Graphs

K. Henderson, T. Eliassi-Rad, C. Faloutsos, L. Akoglu, L. Li, K. Maruhashi, B. A. Prakash, H. Tong

February 17, 2010 
This document was prepared as an account of work sponsored by an agency of the United States government. Neither the United States government nor Lawrence Livermore National Security, LLC, nor any of their employees makes any warranty, expressed or implied, or assumes any legal liability or responsibility for the accuracy, completeness, or usefulness of any information, apparatus, product, or process disclosed, or represents that its use would not infringe privately owned rights. Reference herein to any specific commercial product, process, or service by trade name, trademark, manufacturer, or otherwise does not necessarily constitute or imply its endorsement, recommendation, or favoring by the United States government or Lawrence Livermore National Security, LLC. The views and opinions of authors expressed herein do not necessarily state or reflect those of the United States government or Lawrence Livermore National Security, LLC, and shall not be used for advertising or product endorsement purposes.

This work performed under the auspices of the U.S. Department of Energy by Lawrence Livermore National Laboratory under Contract DE-AC52-07NA27344. 


\title{
MetricForensics: A Multi-Level Approach for Mining Volatile Graphs
}

\author{
Keith Henderson ${ }^{*} \quad$ Tina Eliassi-Rad ${ }^{*} \quad$ Christos Faloutsos $^{\dagger} \quad$ Leman Akoglu $^{\dagger}$

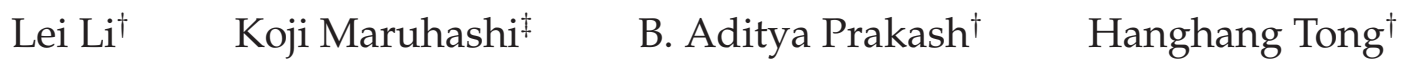

\begin{abstract}
Advances in data collection and storage capacity have made it increasingly possible to collect highly volatile graph data for analysis. Existing graph analysis techniques are not appropriate for such data, especially in cases where streaming or near-real-time results are required. An example that has drawn significant research interest is the cyber-security domain, where internet communication traces are collected and real-time discovery of events, behaviors, patterns and anomalies is desired. We propose MetricForensics, a scalable framework for analysis of volatile graphs. MetricForensics combines a multi-level "drill down" approach, a collection of user-selected graph metrics and a collection of analysis techniques. At each successive level, more sophisticated metrics are computed and the graph is viewed at a finer temporal resolution. In this way, MetricForensics scales to highly volatile graphs by only allocating resources for computationally expensive analysis when an interesting event is discovered at a coarser resolution first. We test MetricForensics on three real-world graphs: an enterprise IP trace, a trace of legitimate and malicious network traffic from a research institution, and the MIT Reality Mining proximity sensor data. Our largest graph has $\sim 3 M$ vertices and $\sim 32 M$ edges, spanning 4.5 days. The results demonstrate the scalability and capability of MetricForensics in analyzing volatile graphs; and highlight four novel phenomena in such graphs: elbows, broken correlations, prolonged spikes, and strange stars.
\end{abstract}

\section{Keywords}

Graph mining, temporal analysis, volatile networks

\section{Introduction}

Given a stream of duration-stamped communication or contact events, how can we find suspicious behavior, anoma-

\footnotetext{
* Lawrence Livermore National Laboratory, \{keith, eliassi\}@1lnl.gov

$\dagger$ Carnegie Mellon University, \{lakoglu, christos, leili, badityap, htong\}@ cs.cmu.edu

‡ Fujitsu Laboratories Ltd., maruhashi.koji@jp.fujitsu.com

This work was performed under the auspices of the U.S. Department of Energy by Lawrence Livermore National Laboratory under contract No. DE-AC52-07NA27344.
}

lous activities, etc? How can we do attribution? For example, in a computer communication graph, we want say to flag the interval that we are under attack, as well as the offending IP address (or addresses).

We define a "volatile graph" to be a stream of durationstamped edges (in its simplest form: $\left\langle v_{s r c}, v_{d s t}\right.$, duration $\rangle$ ), where we assume that there are potentially infinite number of nodes, and that edges may appear and disappear. Examples of volatile graphs include IP-to-IP communication graphs (either at the backbone or at the access-link) as well as physical proximity graphs (e.g., measured by blue-tooth connections).

We introduce METRICFORENSICS which given a volatile graph is able to characterize it and detect interesting events at multiple levels (both temporally and topologically). At the global level, METRICFORENSICS computes and monitors a suite of graph metrics (like the number of active nodes, the first few eigenvalues, their wavelet transforms) at regular intervals. Only when a deviation from usual behavior is flagged, we follow through with a "drill down" approach. We study the offending graph at finer temporal resolution, as well as we examine carefully chosen sub-graphs (community-level) or even individual nodes (local-level) using local metrics and more sophisticated and time-consuming analysis techniques (like ego-net analysis). Thus, we are able to do attribution of the rare event, while maintaining high processing speed.

The contributions of METRICFORENSICS are as follows:

- Effectiveness: METRICFORENSICS spots strange activities, like "elbows" (Section 4.1.1), broken correlations (Section 4.1.2), prolonged spikes (Section 4.1.3), and strange stars (Section 4.3)

- Scalability: All the components of the system are carefully chosen to be not only informative, but also fast to compute (linear on the measures of interest).

- Flexibility and generality: The METRICFORENSICs framework can easily include several modules in addition to the described ones, like spectral analysis, PageRank, etc. Moreover, the method can be applied to any type of volatile graphs (e.g., email/SMS communications).

The outline of the paper is as follows: Section 1 provides an introduction to this work. Section 2 presents an overview of the related work. Section 3 describes our proposed method, METRICFORENSICS. Section 4 presents experimental results on 3 real-world volatile graphs. Lastly, Section 5 provides some concluding remarks. 


\section{Background and Related Work}

We review the related work in this section. It can be categorized into four parts: (1) mining static graphs, (2) mining time-evolving graphs, (3) anomaly detection on graphs and finally (4) mining time series.

Mining Static Graphs. There has been a lot of research work on static graph mining. The work can be grouped into three levels. First, on the graph-level, researchers have studied the statistical properties of some global metrics (e.g., degree distribution, diameter, first eigenvalue, etc) of the whole graph. Representative work includes [3, 13, 7, 26], etc. Next, a lot of work is related to mining the graph on the subgraph level, including frequent substructure discovery [32], graph partition and community detection $[14,16]$. Finally, there has been work on mining the graph on the individual node/edge level as well, including influence propagation [21], link prediction [24], ranking [15], proximity [30] and so on. Note that almost all of the previous work deals with only one of the three levels (graph-level, subgraph level, or node/edge-level). In contrast, our METRICFORENSICS works on all the levels in a "drill down" way.

Mining Dynamic Graphs. More recently, there is an increasing interest in mining time-evolving graphs, such as densification laws and shrinking diameters [23], community evolution [4, 11], dynamic tensor analysis [29] and so on. Again, most of the above analyze only one of the three levels (graph-level, subgraph level, or node/edge-level). Additionally, they often manually choose a single fixed timegranularity to perform the analysis. In contrast, METRICFORENSICS performs a multi-level analysis over time.

Anomaly Detection on Graphs. Most of anomaly detection methods for graph data are based on the minimum description length (MDL) principle [27, 8]. Other representative work includes classification-based methods [25], probabilistic measures [12], spectral methods $[19,18]$ and so on. For anomaly detection on other types of data, please refer to a recent survey [10]. In OddBall [2], they explicitly focus on nodes and interactions are considered implicitly through the neighborhood subgraphs. Unlike most of existing work, we consider edge weights in our anomaly detection method.

Mining Time Series. Representative work in this category includes similarity search [31], click-through rate estimation [1], anomaly detection [28, 22]. Notice that our METRICFORENSICS can naturally incorporate most of these methods into the framework, such as BGP-Lens [28].

\section{MetricForensics}

The flowchart for METRICFORENSICS is depicted in Figure 1. METRICFORENSICS is comprised of three distinct components: (1) a suite of graph metrics, (2) a collection of analysis techniques, and (3) a multi-level approach. We will describe each of these below. But first, we will briefly discuss METRICFORENSICS' data model for representing volatile graphs.

\subsection{Data Model for Volatile Graphs}

Highly volatile graphs, by definition, accumulate massive numbers of vertices and edges over time. However, during a given window of time, only a fraction of these vertices and edges are active. The METRICFORENSICS data model takes advantage of this behavior.

\subsubsection{Snapshot Graphs}

A snapshot graph is defined by its vertices $V_{t}$ and edges $E_{t}$, which are active at time $t$. A snapshot graph can be viewed as an $N \times N$ adjacency matrix representing the graph at time $t$. The dynamic system is then comprised of many such matrices in sequence. Each time a vertex is added or deleted, or an edge appears or disappears, or an edge-weight is changed, a new snapshot graph is generated.

\subsubsection{Summary Graphs}

Due to the high volatility of the data, it is neither computationally feasible nor analytically worthwhile to consider snapshot graphs in isolation. A summary graph summarizes all snapshot graphs a during time period $T$. It is represented by its vertices $V_{T}$ and edges $E_{T}$. Many strategies are available for combining snapshot graphs, including:

- Binary: An unweighted edge $(i, j)$ exists in the summary graph $G_{T}$ if $(i, j)$ exists in at least one snapshot graph during $T$.

- Sum: A weighted edge $w(i, j)$ exists in the summary graph $G_{T}$ if $(i, j)$ exists in any snapshot graph during $T$. Then, $w(i, j)$ is the sum of the weights of edges active at the beginning and during the interval $T$.

- Max: Similar to Sum except that $w(i, j)$ is the maximum value of element $a_{i j}$ in the adjacency matrices of snapshot graphs for time interval $T$.

The frequency with which summary graphs are generated and analyzed is a parameter in METRICFORENSICS, and plays an important role in the multi-level component of the framework (see Section 3.4). Summary graphs can be generated after a fixed number of distinct snapshot graphs or after a fixed period of time. Our experiments demonstrate that the framework works across a reasonably large set of summary graph frequencies, and as a heuristic we tend to choose the frequency so that each summary graph represents no more than 100,000 unique snapshot graphs. See Section 4 for details.

\subsection{Suite of Graph Metrics}

At the heart of METRICFORENSICS is a suite of graph metrics. These metrics are of varying levels of complexity and computational intensity. They are broadly classified into three groups based on their topological granularity: (1) global, (2) community, and (3) local. The framework is readily extendable to include any graph metrics. Moreover, it is not necessary to run all the metrics at all times.

\subsubsection{Global Metrics}

At the coarsest topological level, global metrics generally measure high-level properties of the graph and are largely agnostic to properties at the individual vertices. Table 1 list a subset of METRICFORENSICS' global metrics. Several listed metrics have both unweighted and weighted versions; only the unweighted version is listed here. Most are very fast to 


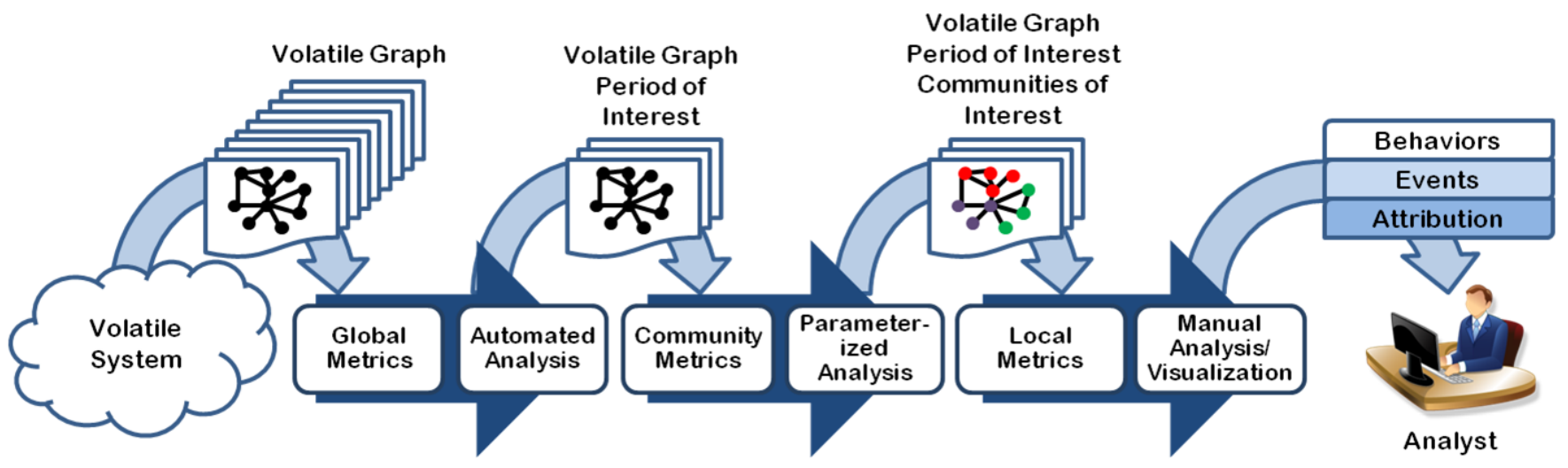

Figure 1. METRICFORENSICS' Flowchart

calculate, scaling linearly with the number of active vertices $\left(N_{T}=\left|V_{T}\right|\right)$ or edges $\left(M_{T}=\left|E_{T}\right|\right)$ in the time interval $T$. Currently, all of our implemented global metrics have complexity at most $O\left(N_{T} \log N_{T}+M_{T}\right)$.

\subsubsection{Community Metrics}

A second collection of metrics examine the graph at its community-structure level. These algorithms are typically more computationally expensive than the global metrics. Many approaches to community discovery in graphs exist [17]. The results presented in Section 4 are based on Cross-Associations (XA) [9]. Regardless of the chosen community discovery algorithm, the metrics are similar.

Some community metrics are static, such as the fraction of vertices in the largest community or the number of communities. Others track changes in community structure, such as the variation of information [20] between successive assignments. If a particular vertex is of interest, then changes in its community can be easily tracked between successive summary graphs.

\subsubsection{Local Metrics}

The final group of metrics focuses on individual vertices. Local metrics often run too slowly to be applied to every vertex in each summary graph. Examples of local metrics include centrality metrics, OddBall [2], and impact metrics (e.g., leaving a single vertex out of the graph and recalculating other metrics to determine the impact of the vertex).

\subsection{Collection of Analysis Techniques}

The second component of METRICFORENSICS is a collection of analysis techniques. Broadly speaking, they fall into three categories: (1) single metric analysis, (2) coupled metric analysis, and (3) non-metric analysis. This component is similar to the suite of metrics in that it can easily accommodate new techniques.

\subsubsection{Single Metric Analysis}

Values for an individual metric across multiple summary graphs can be viewed as a time series. METRICFORENSICS leverages the multitude of time series analysis techniques to identify behaviors, events, and anomalies. For example, an Autoregressive Moving Average Model (ARMA) can be used to identify metric values that are abnormally large or small given recent values. Fourier analysis can identify periodic behavior, such as daily trends in graph properties. Wavelet analysis tools such as BGP-lens [28] identify patterns and anomalies in metric values. Other single-metric tools include lag plots, outlier detection techniques such as Local Outlier Factor [6] and fractal dimension analysis [5].

\subsubsection{Coupled Metric Analysis}

Techniques in this category consider two or more metrics in unison. The simplest such technique is correlation analysis. If $K$ metrics are computed for a series of summary graphs, a $K \times K$ matrix, $C$, can be computed where $C_{i j}$ is say the Pearson correlation between metrics $i$ and $j$. Large values of $\left|C_{i j}\right|$ can identify redundant metrics. If such metrics vary widely in runtime complexity, then the slower ones can be omitted from future calculations. However, it is often useful to retain both metrics; if the computed values of two metrics typically demonstrate high correlation, a sequence of summary graphs that shows lower correlation is identified as an interesting event.

A useful example of coupled metric analysis involves various summary-graph edge-weighing strategies (Section 3.1.2). In particular, if metrics are computed simultaneously on summary graphs constructed using different strategies, such as Sum and Max, the resulting time series data are often highly correlated. In this case, a summary graph for which the metric-values do not demonstrate their typical relationship can be identified as an interesting event.

Other techniques can be applied to coupled metric data, such as outlier detection or clustering. For example, a clustering algorithm like $k$-means can be applied to two time series. Small clusters are labeled as interesting events or behaviors (see Section 4 for details).

\subsubsection{Non-Metric Analysis}

Techniques in this category do not involve the computed metrics 3.2. These techniques are not applied until an interesting event is discovered using the above techniques, but they are often useful for understanding the events. The primary existing techniques in this category are visualization tools and attribute data inspection. 


\begin{tabular}{|c|c|c|c|}
\hline Group & Metric & Time Complexity & Notes \\
\hline Basic Properties & num_vertices & $O(1)$ & Number of active vertices \\
\hline Basic Properties & num_edges & $O(1)$ & Number of active edges \\
\hline Basic Properties & $a v g \_d e g$ & $O(1)$ & Average vertex degree \\
\hline Basic Properties & $a v g \_w g t$ & $O(1)$ & Average edge weight \\
\hline Basic Properties & max_deg & $O\left(N_{T}\right)$ & Number of connected components \\
\hline Connectivity Metrics & num_comps & $O\left(M_{T}\right)$ & Fraction of vertices in the largest component \\
\hline Connectivity Metrics & max_comp & $O\left(M_{T}\right)$ & Number of articulation points \\
\hline Connectivity Metrics & art_pts & $O\left(M_{T}\right)$ & Minimum spanning tree weight \\
\hline Connectivity Metrics & mst_wgt & $O\left(M_{T}\right)$ & Jaccard $\left(V_{T}, V_{T-1}\right)$ \\
\hline Spectral Metrics & $k \_l a m b d a s$ & $O\left(N_{T} k^{2}+M_{T} k\right)$ & Jaccard $\left(E_{T}, E_{T-1}\right)$ \\
\hline Stability Metrics & delta_v & $O\left(N_{T}\right)$ & $O\left(M_{T}\right)$ \\
\hline Stability Metrics & delta_e & $O\left(N_{T}\right)$ & Jaccard coefficient of $k$ highest degree vertices from $T-1$ and $T$ \\
\hline Stability Metrics & delta_topK & &
\end{tabular}

Table 1. A subset of METRICFORENSICS' suite of global graph metrics

METRICFORENSICS currently includes a novel 3D visualization tool that can display summary graphs rapidly and in an informative layout. It highlights vertices with high connectivity, and is used to quickly characterize a sequence of summary graphs that have been identified as interesting. The tool uses position (source vs. target vertex), size, and color to differentiate between vertices according to a userspecified collection of attributes. For example, the size of a vertex can show its degree, while the color can depict the vertex' betweenness centrality. See Figure 3a for a 2D snapshot of a summary graph by our visualization tool.

The second non-metric analysis technique involves inspection and processing of available attribute data. Vertices and edges in volatile graphs can have attributes. In some cases, more detailed attributes may be available at an increased cost of access. These should be retrieved only when necessary. For example, IP communication traces often have at least partial packet contents, but these are usually not available for fast inspection. While it is not feasible to consider every packet in detail, METRICFORENSICS can identify periods of time and sets of edges that may be of interest based on graph metrics or community structure. A user can then apply a full pcap analysis tool to the identified regions.

\subsection{A Multi-Level Approach}

METRICFORENSICS's multi-level approach allows for efficient use of computational resources. Due to the volatile nature of our data (e.g., IP network traces) and the varying complexity of metrics and analysis techniques, it is necessary to rely on lightweight techniques at coarse granularities (both temporally and topologically) to identify regions of interest, and then apply complex algorithms and tools only to interesting regions. METRICFORENSICS uses multiple levels in three distinct dimensions: (1) time, (2) topology, and (3) analysis automation.

The general approach involves performing METRICFORENSICS's metrics and analysis multiple times at different levels, starting with the coarsest and becoming finer at each iteration. Only those time periods identified as interesting in a coarse level are passed down to be analyzed at the next finer level. We generally identify three levels, based on the topo- logical granularity levels (namely, global, community, and local). However, METRICFORENSICS supports any number of levels based on time granularity.

\subsubsection{Time Granularity}

The temporal scale of METRICFORENSICS can be controlled in two ways. (1) the period of time in which summary graphs are analyzed can be adjusted. At the coarsest level, METRICFORENSICS operates on all available data, which in many cases can include streaming data. When an event is detected, only the relevant portion of the data is examined at finer levels. ${ }^{1}$ (2) temporal granularity is adjusted by modifying the interval between summary graphs. At the coarsest level, summary graphs are generated less often than in finer levels. This "drill-down" approach is used to pinpoint changes in behavior of specific vertices (i.e., do attribution).

\subsubsection{Topological Granularity}

The axis of refinement here involves which set of graph metrics are applied. At the coarsest level, only the global topology of the graph is considered. Communities and individual nodes are not generally considered, with the exception of a small number of global statistics that track the identities of high-degree vertices (such as delta_topK in Table 1). The global metrics are scalable and can be computed efficiently on each summary graph. When an event is discovered at this level, the period of interest is passed to the next (finer) level.

At the finer (regional) level, community-level metrics are calculated. By identifying communities that exhibit change, METRICFORENSICS can discard many vertices that have not changed their behavior. This information is subsequently used at the finest level of refinement, where local metrics are computed on vertices in the identified communities.

\subsubsection{Analysis Automation Levels}

The final difference between levels in METRICFORENSICS is the selection of analysis techniques. Some techniques,

\footnotetext{
${ }^{1}$ In a streaming setting, this is accomplished by maintaining a circular buffer that stores a fixed number of recent snapshot graphs.
} 
such as ARMA, are fully automated. These can be applied at any refinement level. Other tools and techniques like visualization and attribute analysis require user interaction and should only be applied to small sets of summary graphs.

\section{Experiments}

We implemented METRICFORENSICS and ran experiments to answer the following questions: (1) Can METRICFORENSICS detect interesting events including anomalies? (2) Do the discovered interesting events tell us something new about the nature of volatile graphs? (3) Is METRICFORENSICS scalable and amenable to real-time (or near real-time) execution?

Table 2 describes the graphs we used in our experiments. ENTP is a IP traffic collected at the perimeter of an enterprise network over 4.5 days in 2007. RMBT is the MIT Reality Mining's blue-tooth connections collected over 12 months. ${ }^{2}$ LBNL is IP traffic collected on an internal enterprise network on $2004 / 12 / 15$ on port \#3. ${ }^{3}$ It includes scanning activities.

\subsection{Experiments at the Global Level}

We discuss some experiments at the global-level of our volatile graphs here. For brevity, we have removed many of results (such as ones from our FFT and wavelet analyses).

\subsubsection{Eigen Analysis}

Figure 2 depicts the two largest eigenvalues in the ENTP summary graphs. In particular, it shows the $\lambda_{1}, \lambda_{2}$ relationship under three different edge-weighing strategies. In the maximum connections strategy, the weight between vertices $i$ and $j$ is equal to the maximum number of simultaneously active connections between $i$ and $j$ during the summary graph's time interval $T$. Under the number of connections strategy, the weight between $i$ and $j$ is equal to the number of active connections between $i$ and $j$ when $T$ started plus the number of connections between $i$ and $j$ during $T$. In the sum of bytes strategy the weight between $i$ and $j$ is the normalized sum of the flow-weights (i.e., number of bytes sent and received) when $T$ started and the weights of flows that occurred between $i$ and $j$ during $T$. Regardless of the summary graphs' edge-weighing strategy, there are special regions where $\lambda_{1}$ is stable and $\lambda_{2}$ is changing, or vice versa. We also observe these special regions in the LBNL trace (see Figure 3), where they are elbow-shaped.

The large eigenvalues of a weighted graph typically correspond to either a single heavy edge, a vertex with high weighted degree, or a component with a large total weight. Thus, when we see a period of time when $\lambda_{1}$ is changing but $\lambda_{2}$ is steady, it is a result of the currently dominant phenomenon changing while the secondary phenomenon is stable (e.g. a single heavy edge changing weight while the structure of the giant component is steady). We refer to this as the "elbow" pattern because it appears as elbowlike structures (Figure 3). A trivial example here is a pair

\footnotetext{
${ }^{2}$ http:reality.media.mit.edu/

${ }^{3}$ http:/ / www.icir.org/enterprise-tracing/download.html
}

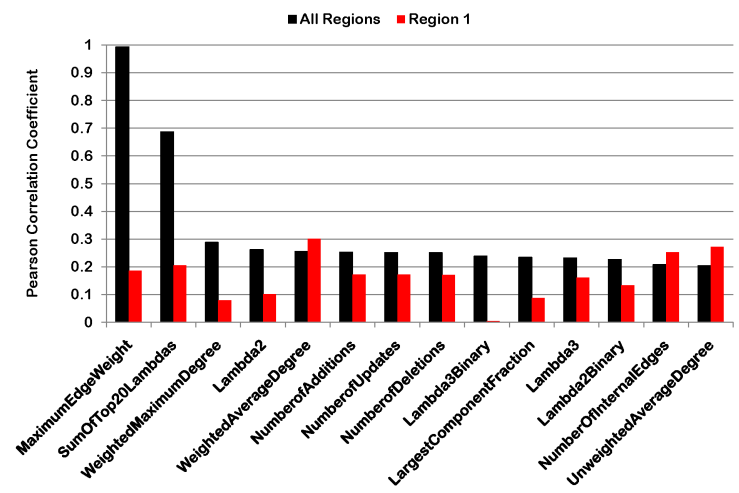

Figure 4. The top-10 graph metrics correlated with $\lambda_{1}$ in the ENTP data. The sharp drop in correlation in Region 1 of Figure 2 is very interesting and depicts a broken correlation.

of heavy edges, $(a, b)$ and $(c, d)$, with $w(c, d)>w(a, b)$ initially. If $w(a, b)$ remains constant and $w(c, d)$ decreases such that eventually $w(c, d)<w(a, b)$, the corresponding eigenvalues will switch so that $\lambda_{1}$ is always correlated with the larger-weight edge. Thus, during the initial period $\lambda_{1}$ tracks the changing $w(c, d)$; but once $w(c, d)<w(a, b), \lambda_{1}$ becomes stable and $\lambda_{2}$ tracks the $(c, d)$ edge.

Depending on the edge-weighting strategy employed, these periods may appear simply as horizontal or vertical sections (Figure 2) or they may appear as elbows (Figure 3). Regardless, the observed behavior is one phenomenon (heavy edge, heavy vertex, or heavy component) that is changing while another phenomenon remains stable.

\subsubsection{Correlation Analysis}

We computed the pairwise Pearson correlation coefficients between values of global metrics. For example, given summary graphs $G_{T_{0}} \cdots G_{T_{t}}$ we computed

$$
r\left(\left[\lambda_{1}^{G_{T_{0}}} \cdots \lambda_{1}^{G_{T_{t}}}\right],\left[\max \_w g t^{G_{T_{0}}} \cdots \max \_w g t^{G_{T_{t}}}\right]\right)
$$

where $\max \_w g t$ is the maximum edge weight. Figure 4 depicts the top-15 most correlated global metrics with $\lambda_{1}$ for the ENTP data. It shows that normally $\lambda_{1}$ is highly correlated with maximum edge weight; however in Region 1 of Figure $2 \mathrm{a}$ (where $\lambda_{1}$ is stable but $\lambda_{2}$ is changing), this correlation disappears. Indeed $\lambda_{1}$ is not correlated with any graph metric in this region. We observed this behavior on other data sets and other eigenvalues. For instance, $\lambda_{2}$ is highly correlated with the fraction of vertices in the largest component, except in regions like Region 2 of Figure 2. In these special regions (where $\lambda_{1}$ is changing but $\lambda_{2}$ is stable), $\lambda_{2}$ is highly correlated with number of additions, number of updates, and number of deletions. We refer to this phenomena as "broken correlations" and observe that there is are meta-level correlations between broken correlations and elbow patterns described above.

\subsubsection{Fractal Dimension Analysis}




\begin{tabular}{|c|c|c|c|c|c|c|}
\hline Data Graph & Observation Time & \# of Source Vertices & \# of Target Vertices & \# of Total Vertices & \# Unique Edges & \# Total Edges \\
\hline ENTP & 4.5 days & $1,748,750$ & $1,733,521$ & $2,928,116$ & $6,597,251$ & $31,855,024$ \\
\hline RMBT & 12 months & 94 & 25,490 & 25,491 & 55,898 & $1,982,576$ \\
\hline LBNL & 1 hour & 3,268 & 2,837 & 3,317 & 15,577 & $9,258,309$ \\
\hline
\end{tabular}

Table 2. Summary of real-world networks used.

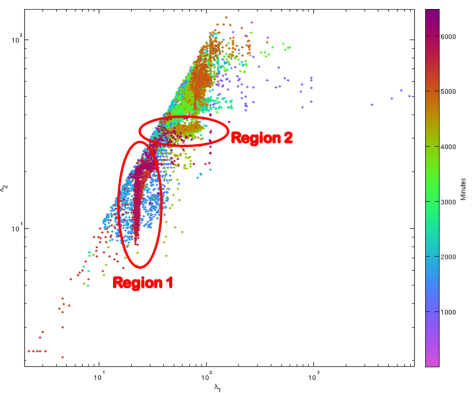

(a) Maximum Connections Strategy

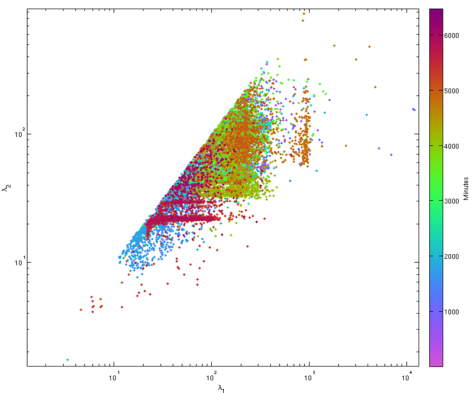

(b) Number of Connections Strategy

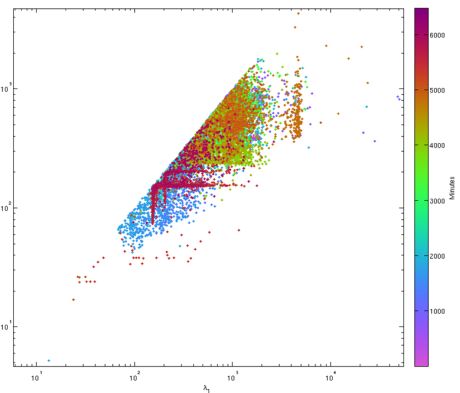

(c) Sum of Bytes Strategy

Figure 2. $\lambda_{2}$ versus $\lambda_{1}$ under various edge-weighing strategies in the ENTP summary graphs (generated every 30 seconds). $x$-axis is $\lambda_{1}$ in log-scale; $y$-axis is $\lambda_{2}$ in log-scale. The color of a dot is the time that it was observed (in minutes): pink/light blue is earlier, red/dark purple is later. Regardless of the summary graphs' edge-weighing strategy, there are interesting regions with elbow patterns where $\lambda_{1}$ is stable and $\lambda_{2}$ is changing, or vice versa.

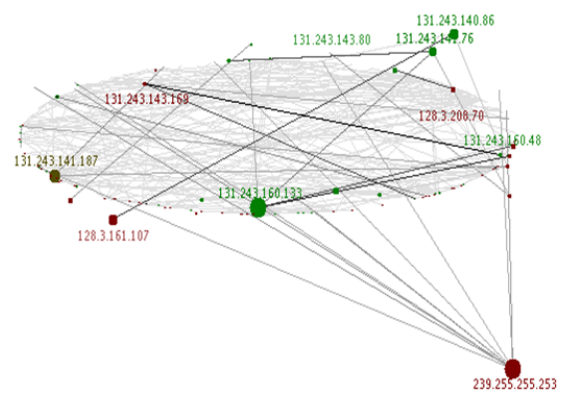

(a) Graph at 2004.12.15 20:06:51.348

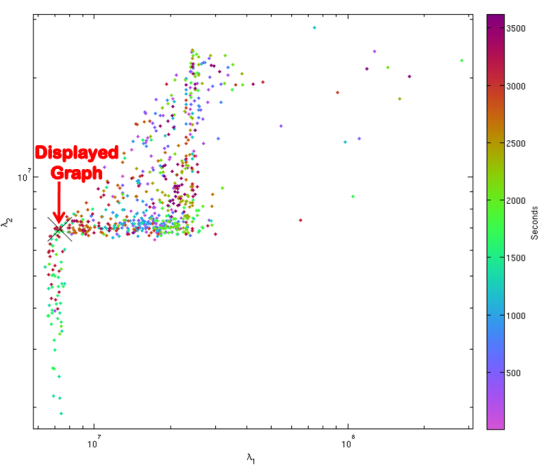

(b) $\lambda_{1}$ vs. $\lambda_{2}$

Figure 3. (a) The LBNL graph at 2004.12.15 20:06:51.348. The vertex colors indicate the recent position of the vertex: source (green) vs. destination (red). The elevation represents the same role but considers the entire history of the vertex. If the vertex is quiet, it slowly moves from green or red back to black; but will not change elevation. The IPs with names either have high weight at 2004.12.15 20:06:51.348 or have had high weight within the last 50 seconds. High weight is defined as $50 \%$ of the current maximum weight in the graph. (b) $\lambda_{2}$ versus $\lambda_{1}$ in the LBNL summary graphs (generated every 5 seconds with sum-of-flows strategy). $x$-axis is $\lambda_{1}$ in log-scale; $y$-axis is $\lambda_{2}$ in log-scale. The elbow patterns occur when the dominant phenomenon and the secondary phenomenon swap roles.

Intuitively, the fractal dimension [5] measures the burstiness of a collection of points. In our case, the points are in 1-dimensional space and correspond to communications at different times. For points that are uniformly distributed in time, the fractal dimension is near the dimensionality of a line, i.e. $D \approx 1$. For points that are all on the same time-tick 
(creating a single burst), the fractal dimension is the dimensionality of a point, $D=0$. The Cantor set (constructed by recursively deleting the middle third of a line segment) has fractal dimension $D=\frac{\log (2)}{\log (3)} \approx 0.63$. Packets due to human behavior typically have fractal dimension somewhere in $[0.7,0.9]$ (with self-similar bursts at different time scales).

We computed the fractal dimensions of several graph metrics on our summary graphs (which can be regarded as a cloud of points on the time axis). Fractal dimensions were calculated for (disjoint) windows of width $w=3$ hours on the ENTP data, $w=5$ minutes on the LBNL data, and $w=10$ days on the RMBT data. The fractal dimension was stable for most time periods, around 0.9 on ENTP and LBNL and around 0.8 on RMBT data. This result suggests that RMBT data is more bursty than others. Interestingly, the fractal dimensions for some metrics (such as number of additions, number of deletions, number of updates, and number of edges connecting to an IP outside the enterprise) suddenly drop to $0.6-0.8$ in some periods on the ENTP data (Figure 5a). Specifically, the fractal dimension of the number of additions suddenly drops down in the early morning on 2007.11.15 (between 6 AM to 9 AM); Figure 5b shows the magnification of that interval, illustrating that the drop is due to a "prolonged spike": activity that has low-volume, but persists for a long time. We also observed this phenomena with wavelet analysis and the BGP-lens package [28], but we omit the wavelet analysis, for lack of space.

\subsection{Experiments at the Community Level}

When at the global-level a summary graph is flagged as interesting, the next step is to analysis the flagged summary graph at their community-level. Figure 6a depicts the pairwise plot of $\lambda_{1}$ and the fraction of source nodes in the largest XA row-group (a.k.a. row-group fraction) during Region 1 from Figure 2. The points are clustered by $k$-means with $k=8$, which produces five singleton clusters, one cluster of size 6, one cluster of size 37, and another one of size 352 . While the singleton clusters and the small cluster of 6 are detectable from $\lambda_{1}$, the larger clusters have nearly identical centroids in $\lambda_{1}$ but are separable by their XA row-group assignments. For those Summary Graphs in the cluster of size 37, vertices in the largest row-group are marked as suspicious. Figure $6 \mathrm{~b}$ shows an exponential moving average of the XA row-group variation-of-information (where lower values indicate more stable community structures) in LBNL. As pointed out by the red arrow, there is a noticeable dip between 750 and 1100 seconds from the start of the hour when communities are very stable. Figure $6 c$ illustrates the XA row group variation of information against the same measure on column groups. We observe that they are correlated, but there are also instances for which one is abnormally high or low given the other (i.e., points in the dashed red circles). These indicate that there vertices whose rowgroups are changing but not their column-groups (i.e., they are changing their behavior as source vertices but not as target vertices), and vice versa.

\subsection{Experiments at the Local Level}

When METRICFORENSICS detects interesting events in the given stream of summary graphs, it can zoom into those interesting graphs and perform more rigorous analysis. In such cases, the main goal is to find interesting (extreme, outlying, suspicious) vertices in a graph. To do so, features from the neighborhood of vertices are extracted. In particular, given a vertex, its neighbors, and the connections between them (a.k.a. the induced 1-step subgraph of the vertex or the egonet), METRICFORENSICS can employ a local-level analysis tool like oddball [2]. Oddball computes the number of edges, the total weight of edges, etc and defines the vertices as points in a multi-dimensional feature space, in which it looks for anomalies.

Figure 7 a shows the number of edges versus the number of vertices in the egonets of RMBT. Each point in the scatter plot corresponds to a particular vertex. Here, the dashed blue line with slope 2 corresponds to cliques for which the number of edges is $O\left(N^{2}\right)$ and similarly the dashed black line corresponds to stars. We observe that most of the points lie on the blue line which indicates that a vast majority of vertices have neighborhoods that look like a clique. For RMBT, this is intuitive; all blue-tooth devices in a specific region will "see" each other, and hence form cliques. On the other hand, we also observe a second cluster of nodes that are neither cliques nor stars. The outlier points here are the black and the blue triangles, which indicate two big strange stars. Figure $7 \mathrm{~b}$ shows the total edge-weight versus degree in RMBT. Here, the weights denote the number of times two devices where close enough to connect to each other. We again observe that vertices form two clusters. The two triangles shown in the circle are the same points as the ones discussed earlier in Figure 7a. These vertices not only form very big star-like structures, but also their total edge-weights are lower than expected. We refer to these as "strange stars."

We performed similar analysis on the LBNL graph, where the vast majority of the vertices form star-like structures. This is intuitive since our LBNL data is a sample of the network traffic over a limited amount of time ( $\approx 1$ hour), so have partial information about the interaction between all vertices. Figure $7 \mathrm{c}$ and $7 \mathrm{~d}$ show the total weight versus the number of edges in the egonets of nodes in the LBNL graph without and with scanning activities, respectively. Here the weights denote the total number of packets sent between pairs of machines. On each plot, we show the top 100 anomalies we detected using a simple metric of the distance from the fitting line. We note that, we were able to detect nonscanner vertices that sent much fewer packets than expected compared to the number of machines they connected with (point shown in square on the figures) as well as detect scanners with a similar behavior: fewer packets than the norm over links (points shown in circle on the same figures).

For the ENTP data, we observed a massive increase (of 10x) in communications around 9 AM on 2007.11.12. Looking at the flow data, we observed a pair of machines that opened over $10 \mathrm{~K}$ connections in about a minute on a BitTorrent related port. Moreover in early morning hours of 2007.11.13, we observed an order of magnitude increase in $\lambda_{1}$ but didn't see a corresponding jump in $\lambda_{1}$ computed on the unweighted summary graph. This was a case where looking at traffic volume alone could not detect the single 


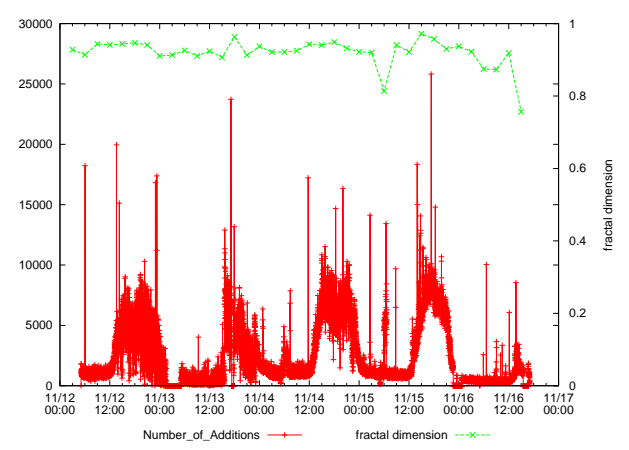

(a) \# Additions

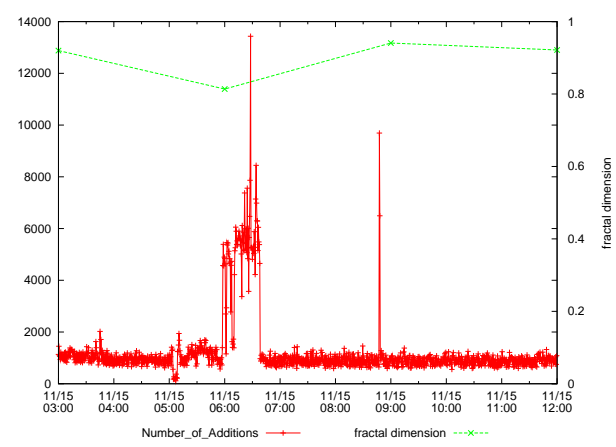

(b) \# Additions on the Magnified X-axis Scale

Figure 5. Fractal Dimension Analysis on ENTP data (best viewed in color). Bi-plots: Number of updates (in red) and fractal dimension $D$ (in green), versus time-stamp. (a) the full interval of analysis - note the drop of fractal dimension around 6 AM on 2007.11.15. (b) shows the magnification of the suspicious region, which has a "prolonged spike" (low volume, but prolonged activity-level).

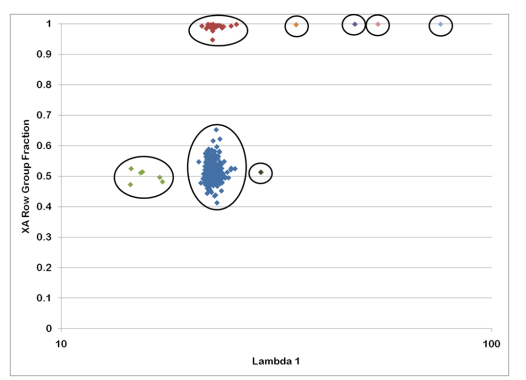

(a) ENTP: k-Means Clusters for Row-Group Fraction vs. $\lambda_{1}$

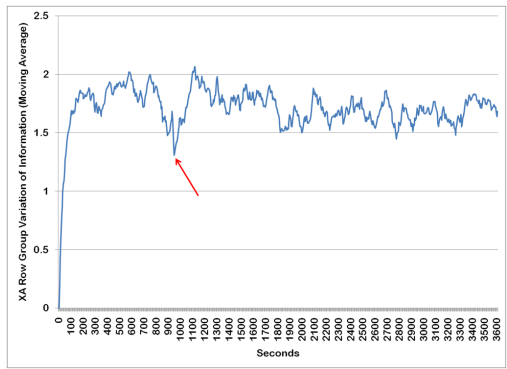

(b) LBNL: Row-Group's Variation-of-Information

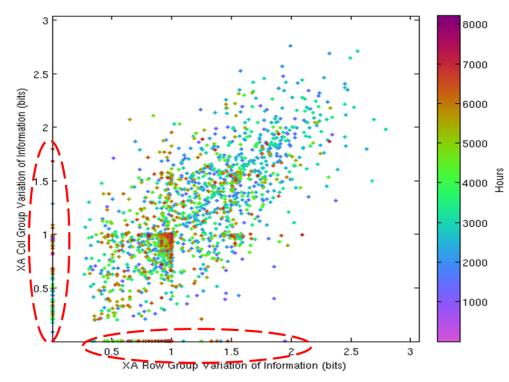

(c) RMBT: Variation-of-Information on Column- vs. Row-Group

Figure 6. Community-Level Experiments with Cross-Associations (XA). (a) During Region 1 (shown in Figure 2a), ENTP has behaviors that can be detected using XA (red cluster) but not using other metrics $\left(\lambda_{1}\right.$ shown). (b) For the LBNL data, there is a pronounced increase in community stability for about 5 minutes. (c) RMBT includes times where source vertices form stable communities but targets do not, and vice versa as encircled by the dashed red ovals.

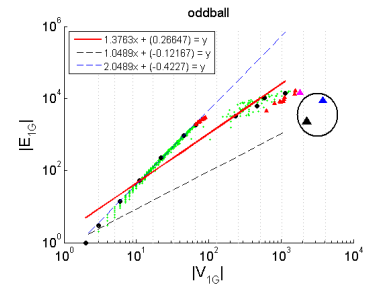

(a) RMBT: $E_{e}$ vs. $N_{e}$

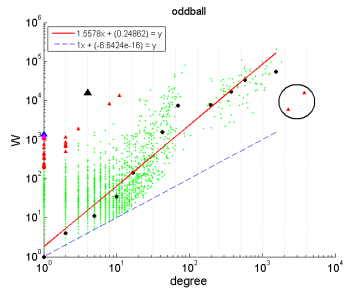

(b) RMBT: $W$ vs. degree

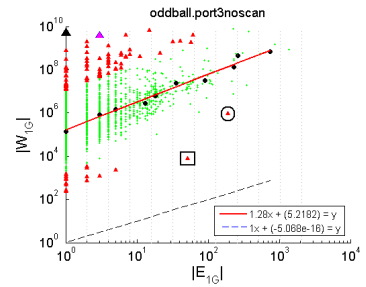

(c) LBNL': $W_{e}$ vs. $E_{e}$

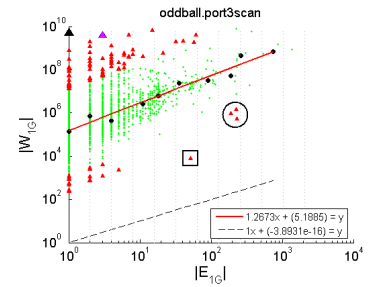

(d) LBNL: $W_{e}$ vs. $E_{e}$

Figure 7. Local-Level Experiments with OddBall. LBNL' is the LBNL data without scanning activity. For a given vertex, $W$ and Degree are its sum of edge-weights and its number of neighbors, respectively. $N_{e}$ and $E_{e}$ are the number of vertices and edges in a vertex' egonet, respectively. $W_{e}$ is the total weight of all edges in a vertex' egonet. The vertices circled have strange star-like neighborhoods.

heavy edge that caused $\lambda_{1}$ to spike for several minutes. For brevity, we omit the plots for these.

\section{Conclusions}

Volatile graphs (such as IP-to-IP communication graphs) 
are becoming more ubiquitous in network science applications. Challenges associated with mining of such graphs include dealing with an ever-changing graph, analysis in streaming or real-time fashion, and analysis at multiple temporal and topological granularities. In this paper, we presented METRICFORENSICS: a multi-level framework for mining volatile graphs that addresses the aforementioned challenges. Its strong points are the following:

- MetricForensics is effective, capable of spotting suspicious patterns like the "elbow" pattern, prolonged spikes, broken correlations, and more.

- It is scalable, with carefully chosen operations, fast-tocompute components (eigenvalues, wavelets, etc), and global-to-local architecture, for efficient runtimes.

- It is flexible, general and extensible, with room for many more components, in addition to ones used (fractal analysis, oddBall, wavelets, etc.)

We illustrated the generality and applicability of METRICFORENSICS on several large (up to $\sim 32 \mathrm{M}$ edges) realworld volatile graphs.

\section{References}

[1] D. Agarwal, A. Z. Broder, D. Chakrabarti, D. Diklic, V. Josifovski, and M. Sayyadian. Estimating rates of rare events at multiple resolutions. In $K D D$, pages 16-25, 2007.

[2] L. Akoglu, M. McGlohon, and C. Faloutsos. OddBall: Spotting anomalies in weighted graphs. In PAKDD, 2010.

[3] R. Albert, H. Jeong, and A.-L. Barabasi. Diameter of the world wide web. Nature, (401):130-131, 1999.

[4] L. Backstrom, D. P. Huttenlocher, J. M. Kleinberg, and $X$. Lan. Group formation in large social networks: membership, growth, and evolution. In $K D D$, pages 44-54, 2006.

[5] A. Belussi and C. Faloutsos. Estimating the selectivity of spatial queries using the 'correlation' fractal dimension. In $V L D B$, pages 299-310, Zurich, Switzerland, Sept. 1995.

[6] M. M. Breunig, H.-P. Kriegel, R. T. Ng, and J. Sander. LOF: Identifying density-based local outliers. In SIGMOD, pages 93-104, 2000.

[7] A. Broder, R. Kumar, F. Maghoul1, P. Raghavan, S. Rajagopalan, R. Stata, A. Tomkins, and J. Wiener. Graph structure in the web: experiments and models. In WWW, 2000.

[8] D. Chakrabarti. Autopart: Parameter-free graph partitioning and outlier detection. In $P K D D$, pages 112-124, 2004.

[9] D. Chakrabarti, S. Papadimitriou, D. Modha, and C. Faloutsos. Fully automatic cross-associations. In KDD, pages 79-88, 2004.

[10] V. Chandola, A. Banerjee, and V. Kumar. Anomaly detection: A survey. ACM Comput. Surv., 41(3), 2009.

[11] Y. Chi, X. Song, D. Zhou, K. Hino, and B. L. Tseng. Evolutionary spectral clustering by incorporating temporal smoothness. In KDD, pages 153-162, 2007.
[12] W. Eberle and L. B. Holder. Mining for structural anomalies in graph-based data. In DMIN, 2007.

[13] M. Faloutsos, P. Faloutsos, and C. Faloutsos. On power-law relationships of the internet topology. SIGCOMM, pages 251-262, Aug-Sept. 1999.

[14] G. Flake, S. Lawrence, C. L. Giles, and F. Coetzee. Self-organization and identification of web communities. IEEE Computer, 35(3), Mar. 2002.

[15] F. Geerts, H. Mannila, and E. Terzi. Relational link-based ranking. In $V L D B$, pages 552-563, 2004.

[16] D. Gibson, J. Kleinberg, and P. Raghavan. Inferring web communities from link topology. In Hypertext, pages 225-234, New York, 1998.

[17] K. Henderson, T. Eliassi-Rad, S. Papdimitriou, and C. Faloutsos. HCDF: A hybrid community discovery framework. In SDM, 2010.

[18] S. Hirose, K. Yamanishi, T. Nakata, and R. Fujimaki. Network anomaly detection based on eigen equation compression. In KDD, pages 1185-1194, 2009.

[19] T. Idé and H. Kashima. Eigenspace-based anomaly detection in computer systems. In $K D D$, pages 440-449, 2004.

[20] B. Karrer, E. Levina, and M. E. J. Newman. Robustness of community structure in networks. Phys. Rev. E, 77(046119), 2008.

[21] D. Kempe, J. Kleinberg, and E. Tardos. Maximizing the spread of influence through a social network. In KDD, pages 137-146, Washington, DC, 2003.

[22] J.-G. Lee, J. Han, and X. Li. Trajectory outlier detection: A partition-and-detect framework. ICDE, pages 140-149, April 2008.

[23] J. Leskovec, J. M. Kleinberg, and C. Faloutsos. Graphs over time: densification laws, shrinking diameters and possible explanations. In KDD, pages 177-187, 2005.

[24] D. Liben-Nowell and J. Kleinberg. The link prediction problem for social networks. In Proc. CIKM, 2003.

[25] C. Liu, X. Yan, H. Yu, J. Han, and P. S. Yu. Mining behavior graphs for "backtrace" of noncrashing bugs. In SDM, 2005.

[26] M. E. J. Newman. The structure and function of complex networks. SIAM Review, 45:167-256, 2003.

[27] C. C. Noble and D. J. Cook. Graph-based anomaly detection. In KDD, pages 631-636, 2003.

[28] B. A. Prakash, N. Valler, D. Andersen, M. Faloutsos, and C. Faloutsos. BGP-lens: patterns and anomalies in internet routing updates. KDD, pages 1315-1324, 2009.

[29] J. Sun, D. Tao, and C. Faloutsos. Beyond streams and graphs: dynamic tensor analysis. In $K D D$, pages 374-383, 2006.

[30] H. Tong, C. Faloutsos, and J.-Y. Pan. Random walk with restart: Fast solutions and applications. Knowledge and Information Systems: An International Journal (KAIS), 2008.

[31] Q. Wang, V. Megalooikonomou, and C. Faloutsos. Time series analysis with multiple resolutions. Inf. Syst., 35(1):56-74, 2010.

[32] D. Xin, J. Han, X. Yan, and H. Cheng. Mining compressed frequent-pattern sets. In $V L D B$, pages 709-720, 2005. 